\title{
Doppelsieg für IL-17A-Antikörper gegen Psoriasis
}

\section{Hier steht eine Anzeige.}

\author{
Plaque-Psoriasis lässt sich mit dem Interleukin(IL)-17A-Antikörper \\ Ixekizumab wirksamer behandeln als mit Placebo und auch als mit \\ Etanercept. Das haben jetzt zwei unabhängige Phase-3-Studien des \\ UNCOVER-Programms ergeben.
}

A n den randomisierten doppelblinden Studien UNCOVER-2 und -3 waren 1.224 respektive 1.346 Erwachsene mit chronischer Plaque-Psoriasis beteiligt. Sie hatten im Psoriasis Area and Severity Index (PASI) mindestens 12 (im Mittel 20) Punkte, ihre Körperoberfläche war zu mindestens $10 \%$ (durchschnittlich 27\%) befallen, und die Gesamtbeurteilung des Arztes lautete auf mittelschwere bis schwere Psoriasis (static Physician Global Assessment, sPGA $\geq 3$ ). In beiden Studien wurden die Patienten im Verhältnis 2:2:2:1 folgenden Therapiearmen zugeteilt: Ixekizumab $80 \mathrm{mg}$ alle zwei Wochen beziehungsweise alle vier Wochen (jeweils nach einer initialen Dosis von $160 \mathrm{mg}$ ), Etanercept $50 \mathrm{mg}$ zweimal pro Woche oder Placebo. Primäre Studienendpunkte nach zwölf Wochen waren eine Verbesserung um mindestens $75 \%$ im PASI und der Anteil der Patienten mit einem sPGA von $0-1$.

In beiden Endpunkten waren die Ixekizumab-Regime den Vergleichstherapien signifikant überlegen: Einen PASI 75 erreichten mit dem IL-17AHemmer bei zweiwöchentlicher Gabe $89,7 \%$ und $87,3 \%$ der Patienten (UNCOVER-2 bzw. -3) und bei vierwöchentlicher Applikation 77,5\% und $84,2 \%$. Mit Etanercept war dies nur bei 41,6 \% beziehungsweise $53,4 \%$ der Fall, die entsprechenden Raten unter Placebo betrugen $2,4 \%$ und 7,3\%. Der Vorteil der Antikörpertherapie gegenüber dem TNF- $\alpha$-Hemmer war bereits nach einer Woche zu erkennen. Mit Ixekizumab kam es bei vielen Patienten sogar zu einem vollständigen Verschwinden der Plaques (PASI 100): bei Therapieintervallen von zwei Wochen bei $41 \%$ und $38 \%$, bei Abständen von vier Wochen bei $31 \%$ und $35 \%$. Unter Etanercept wurden dagegen nur $5 \%$ und $7 \%$ der Patienten plaquefrei, unter Placebo $0,6 \%$ und $0 \%$. Entsprechend deutlich waren auch die Unterschiede im

sPGA. Werte von 0 oder 1 erreichten mit Ixekizumab alle zwei Wochen $83,2 \%$ und $80,5 \%$, mit Ixekizumab alle vier Wochen $72,9 \%$ und $75,4 \%$ der Patienten, aber nur $36,0 \%$ und $41,6 \%$ mit Etanercept sowie 2,4\% und $6,7 \%$ mit Placebo.

Parallel zu den klinischen Verbesserungen unter dem IL-17A-Hemmer wurden eine Zunahme der Lebensqualität und ein Rückgang des Juckreizes festgestellt. In diesen sekundären Endpunkten war der Antikörper ebenfalls signifikant wirksamer als die Vergleichsbehandlungen.

Sicherheitsprofil und Nebenwirkungen von Ixekizumab waren vergleichbar mit denen aus den Phase-II-Studien und denen von Etanercept. Mit dem Antikörper behandelte Patienten litten allerdings häufiger an Infektionen (jeweils $26 \%$ vs. $22 \%$ mit Etanercept und $21 \%$ mit Placebo), meistens handelte es sich um Nasopharyngitiden und Infekte der oberen Atemwege. Gravierende Nebenwirkungen traten in allen vier Gruppen gleich selten auf (jeweils 1,9\%), Todesfälle gab es keine.

Fazit: Die selektive Ausschaltung von Interleukin 17A durch einen hochaffinen Antikörper könnte für Psoriasispatienten eine neue und wirksame Therapieoption sein, so die Autoren. Sie räumen allerdings ein, dass im Hinblick auf Wirkung und Sicherheit noch die Langzeitergebnisse der Studien abzuwarten sind.

Dr. Beate Schumacher

Griffiths CEM et al. Comparison of ixekizumab with etanercept or placebo in moderate-tosevere psoriasis (UNCOVER-2 and UNCOVER-3): results from two phase 3 randomised trials. Lancet 2015; 386: 541-51 